\title{
Heterotic - Type I Duality in Four Dimensions in the Presence of Anomalous $U(1)$ 's
}

\author{
Stéphane Lavignac* \\ Physikalisches Institut, Universität Bonn \\ Nussallee 12, D-53115 Bonn, Germany \\ E-mail: liavignac@th.physik. uni-bonn. dei
}

ABSTRACT: In this talk, we review the properties of anomalous $U(1)$ 's in $D=4, N=1$ type IIB orientifolds and investigate the pertubative validity of $N=1$ heterotic-type I duality in four dimensions.

\section{Introduction}

In the last few years, the development of the orientifold techniques ploration of a new class of $D=4, N=1$ string compactifications with promising phenomenolo-

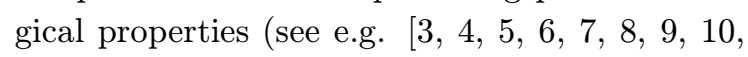
111', 112in). These new vacua, which contain both open and unoriented closed strings, are expected to have heterotic duals in view of the postulated heterotic-type I duality in ten dimensions [1] 13 in]. One of the most striking features of these orientifold models, however, is the presence of anomalous abelian gauge symmetries which show many differences with the heterotic anomalous $U(1)$. In particular, their gauge groups may contain several anomalous $U(1)$ 's, with non-universal anomalies canceled by a generalized Green-Schwarz mechanism involving several antisymmetric tensors, and moduli-dependent Fayet-Iliopoulos terms.

One may thus ask whether this picture is consistent with duality.

In this talk, we first give a detailed description of anomalous $U(1)$ 's in orientifolds, with a particular attention to the scales of the gauge boson masses and Fayet-Iliopoulos terms. Then we use these results to discuss the validity of duality on specific examples where a candidate heterotic dual is known.

*This talk is based on work made in collaboration with Z. Lalak and H.P. Nilles [1]'

\section{Anomalous $U(1)$ 's in $D=4, N=1$ heterotic compactifications}

Let us first recall some facts about anomalous $U(1)$ 's in $D=4, N=1$ compactifications of the heterotic string. The gauge group of such vacua often possesses several abelian factors, one combination of which (denoted by $U(1)_{X}$ in the following) may be anomalous. Its anomalies are harmless, however, since they are compensated for by a four-dimensional version [1] Schwarz mechanism [1] ${ }_{1}^{1}$ - $]$ which ensures the consistency of the underlying $D=10$ string theory. This mechanism relies on the presence of an antisymmetric tensor with the requisite couplings to cancel the anomaly; since there is only one such tensor $B_{\mu \nu}$ in heterotic vacua (the fourdimensional remnant of the antisymmetric tensor from the $D=10$ supergravity multiplet), there can be at most one anomalous $U(1)$. In its supersymmetric formulation [1] $\left.{ }_{1}^{1}\right]$, the Green-Schwarz mechanism ist most commonly described in terms of the pseudoscalar dual of $B_{\mu \nu}$, the model-independent axion that belongs to the dilaton supermultiplet. The anomalies are then canceled by a shift of the axion under a $U(1)_{X}$ gauge transformation. Note that this requires universal (gaugegroup independent) ratios of the anomalies:

$$
\frac{C_{A}}{k_{A}}=\frac{C_{g}}{12}
$$

where $k_{A}$ is the Kac-Moody level of the gauge group factor $G_{A}, C_{A}=2 \operatorname{Tr}\left(T^{A} T^{A} X\right)$ is the 
coefficient of the $U(1)_{X} G_{A} G_{A}$ gauge anomaly (there is an additional symmetry factor for the cubic $U(1)_{X}$ anomaly, $\left.C_{X}=\frac{2}{3} \operatorname{Tr}\left(X^{3}\right)\right)$, and $C_{g}=\operatorname{Tr} X$ is the coefficient of the mixed $U(1)_{X^{-}}$ gravitational anomaly.

The anomalous gauge boson acquires a mass of the order of the string scale through its GreenSchwarz coupling to the axion and, as a consequence of the anomaly, a Fayet-Iliopoulos term $\xi^{2}$ is generated at the one-loop level. A string computation [1 $\left.1 \overline{6}_{1}^{\prime}\right]$ shows that $\xi^{2}$ is tied to the string scale by a model-dependent coefficient:

$$
\xi^{2}=\frac{\operatorname{Tr} X}{192 \pi^{2}} M_{S t r}^{2}
$$

In the original vacuum, supersymmetry is broken by the Fayet-Iliopoulos term; however, there generally exist "shifted vacua" in which the vevs of some fields $\Phi_{\alpha}$ charged under $U(1)_{X}$ compensate for $\xi^{2}$, thus restoring supersymmetry. A combination of the $S$ and the $\Phi_{\alpha}$ chiral superfields is then absorbed by the anomalous vector multiplet and disappears from the massless spectrum, while the orthogonal combination yields the lowenergy dilaton supermultiplet. This vacuum shift generally triggers further breakdown of the gauge group $\left[1 \overline{i_{1}}\right]$.

\section{Anomalous $U(1)$ 's in $D=4, N=1$ orientifolds}

As stressed in the introduction, $D=4, N=1$ type IIB orientifolds show a very different pattern of anomaly cancellation. The gauge group of such vacua may contain more than one anomalous $U(1)$. Their anomalies are not universal in the sense of Eq. (2.1) and are compensated for by a generalized version of the Green-Schwarz mechanism, which involves several antisymmetric tensors [1] ${ }^{1}$ ]. Also, a string computation [1 $\left.\overline{9}_{1}^{\prime}\right]$ has shown that no Fayet-Iliopoulos term is generated at the one-loop level. While this result has been obtained in a specific vacuum (the $Z_{3}$ orientifold of Ref. [3i] ), it is believed to hold in a larger class of models, since it is related to tadpole cancellation.

The mechanism of anomaly cancellation in $T^{6} / Z_{N}$ and $Z_{M} \times Z_{N}$ type IIB orientifolds has been studied in Ref. [201]'. Let us summarize here their results. In addition to the antisymmetric tensor $B_{\mu \nu}$ from the untwisted sector, these models contain several twisted antisymmetric tensors $B_{k \mu \nu}, k=1 \ldots M$ living at the fixed points of the underlying orbifold. Those twisted antisymmetric tensors couple ${ }^{1}$ to the Yang-Mills ChernSimons forms:

$$
\partial^{\mu} B_{k}^{\nu \rho} \omega_{\mu \nu \rho}^{A Y M}
$$

and to the field strength of the abelian factors $U(1)_{i}, i=1 \ldots N$ present in the gauge group:

$$
\epsilon_{\mu \nu \rho \sigma} B_{k}^{\mu \nu} F_{i}^{\rho \sigma}
$$

The Green-Schwarz terms $(\overline{3} .2 \overline{2})$, together with (3.13), generate the tree diagrams which cancel against the anomalous triangle graphs. A striking difference with the heterotic case is that there are no Green-Schwarz couplings for $B_{\mu \nu}$ - implying that the dilaton superfield does not play any role in anomaly cancellation [20

In a supersymmetric description, the pseudoscalar duals $a_{k}$ of the twisted antisymmetric tensors lie in the same chiral multiplets as the blowing-up modes $m_{k}$ associated with the singularities of the orbifold:

$$
\left.M_{k}\right|_{\theta=\bar{\theta}=0}=m_{k}+i a_{k}
$$

Performing a duality transformation on the couplings $(3.1)$, we obtain the following expression for the gauge kinetic function:

$$
f_{A}=f_{p}+\sum_{k} c_{A}^{k} M_{k}
$$

where $f_{p}$ depends linearly on the untwisted moduli $\left(f_{p}=S\right.$ for gauge groups coming from 9branes [iqu] ) and the $c_{A}^{k}$ are model-dependent coefficients. Similarly, the couplings (3.2) can be rewritten as

$$
\partial_{\mu} a_{k} A_{i}^{\mu}
$$

This tells us that the Kähler potential for the $M_{k}$ fields takes the generic form:

$$
K=K\left(\left\{M_{k}+\bar{M}_{k}-2 \sum_{i=1}^{N} \delta_{i}^{k} V_{i}\right\}\right)
$$

\footnotetext{
${ }^{1}$ More precisely, the antisymmetric tensors living at a given fixed point of the orbifold couple only to the gauge fields coming from D-branes whose worldvolume contains this fixed point.
} 
where the Green-Schwarz parameters $\delta_{i}^{k}$ are model-dependent coefficients as well ${ }^{2}$. Gauge invariance then implies that, under a $U(1)_{i}$ transformation with gauge parameter $\Lambda_{i}$, the $M_{k}$ undergo a shift:

$$
M_{k} \rightarrow M_{k}+i \delta_{i}^{k} \Lambda_{i}
$$

while the dilaton, as well as the other untwisted moduli, remains unshifted. The variation of the gauge kinetic terms induced by (3.7i) then cancels the mixed gauge anomalies, which show the nonuniversal structure:

$$
C_{A}^{i}=8 \pi^{2} \sum_{k} c_{A}^{k} \delta_{i}^{k}
$$

Mixed gravitational anomalies are canceled in a similar fashion.

In order to determine the Fayet-Iliopoulos terms and abelian gauge boson masses, one first needs to find the anomalous combinations of the $N U(1)$ factors. This is done by diagonalizing the Green-Schwarz terms

$$
2 \sum_{i, k} g_{i} \widetilde{\delta}_{i}^{k} \partial_{\mu} a_{k} A_{i}^{\mu}
$$

as obtained from $\left(\underline{3}_{-}^{-} \cdot \bar{b}\right)$, where the $a_{k}$ and $A_{i}^{\mu}$ fields have been canonically normalized, $g_{i}=$ $\left\langle\operatorname{Re} f_{i}\right\rangle^{-1 / 2}$ is the gauge coupling of $U(1)_{i}$ and $\widetilde{\delta}_{i}^{k}=\sqrt{2} \sum_{k} K_{k l}^{1 / 2} \delta_{i}^{l}$ are the moduli-dependent Green-Schwarz couplings $\left(K_{k l}=\partial^{2} K / \partial M_{k} \partial \bar{M}_{l}\right.$ denotes the Kähler metric for the $M_{k}$ fields). The "diagonalization" of (3.9i) involves a rotation $R^{V}$ on the gauge bosons and a rotation $R^{M}$ on the pseudoscalars $a_{k}$ :

$$
\sum_{i, k} R_{p i}^{V} g_{i} \widetilde{\delta}_{i}^{k} R_{q k}^{M}=\mu_{p} \delta_{p q}
$$

One can always choose $R^{V}$ and $R^{M}$ in such a way that the first $r$ "eigenvalues" $\mu_{1}, \ldots \mu_{r}$ are nonzero. In terms of the redefined fields $a_{p}^{\prime}=$ $\sum_{k} R_{p k}^{M} a_{k}$ and $A_{p}^{\prime \mu}=\sum_{i} R_{p i}^{V} A_{i}^{\mu}$, the Green-Schwarz couplings $\left(3^{-} \cdot 9^{i}\right)$ read

$$
\sum_{p=1}^{r} \mu_{p} \partial_{\mu} a_{p}^{\prime} A_{p}^{\prime \mu}
$$

\footnotetext{
${ }^{2}$ The coefficients $c_{A}^{k}$ and $\delta_{i}^{k}$ were determined in [20] from the study, of anomaly cancellation, and explicitly computed in [211].
}

Eq. (B. $\overline{1} \overline{1})$ tells us that there are exactly $r$ anomalous $U(\overline{1})$ 's. Indeed, only the $r$ gauge bosons $A_{p=1 \ldots r}^{\prime \mu}$ have Green-Schwarz couplings, and each of them couples to a different axion-like field $a_{p}^{\prime}$ whose shift compensates for the anomalies of the associated $U(1)$. The $N-r$ orthogonal combinations $A_{p=r+1 \ldots N}^{\prime \mu}$, on the other hand, correspond to anomaly-free $U(1)$ 's. This can be checked explicitely by redefining the charges accordingly to the gauge bosons, $Y_{p}^{\prime}=\sum_{i} R_{p i}^{V} g_{i} Y_{i} / g$ (with $\left.g=\sqrt{\sum_{i} g_{i}^{2} / N}\right)$ and by computing the anomalies in the new basis. Note that the anomalous gauge bosons are defined in an unambiguous way by Eq. (i. $\overline{1} \overline{1})$ (up to possible degeneracies among the $\mu_{p}$ ), while the anomaly-free gauge bosons can be rotated at will.

Having identified the anomalous $U(1)$ 's, we are now ready to determine the gauge boson masses and Fayet-Iliopoulos terms. Eq. ( $\left(\bar{\beta} . \overline{1} \overline{1}_{1}^{\prime}\right)$ tells us that the anomalous gauge boson $A_{p}^{\prime \mu}$ acquires a mass $\mu_{p}$ by absorbing the pseudoscalar $a_{p}^{\prime}$. The supersymmetric version of this statement is that the chiral superfield $M_{p}^{\prime}$ and the anomalous vector multiplet $V_{p}^{\prime}$ combine to form a massive vector multiplet, with $m_{p}^{\prime}$ as a scalar component, and $a_{p}^{\prime}$ providing the longitudinal degree of freedom of the vector component $A_{p}^{\prime \mu}$. The masses $\mu_{p}$, together with the rotations $R^{M}$ and $R^{V}$, can be computed by diagonalizing either the gauge boson or the scalar mass matrix (both of them are obtained from the expansion of the Kähler potential $(\overline{3} \cdot \overline{6}))$. Choosing this last possibility, we define $R^{\bar{M}}$ as the rotation which diagonalizes $M_{k l}^{2}=\sum_{i} g_{i}^{2} \widetilde{\delta}_{i}^{k} \widetilde{\delta}_{i}^{l} M_{P l}^{2}$. We then obtain an expression for the masses:

$$
\mu_{p}^{2}=\sum_{i} g_{i}^{2} \bar{\delta}_{i}^{p} \bar{\delta}_{i}^{p} M_{P l}^{2} \quad \bar{\delta}_{i}^{p}=\sum_{k} R_{p k}^{M} \widetilde{\delta}_{i}^{k}
$$

and for the anomalous combinations of the gauge bosons:

$$
A_{p}^{\prime \mu}=\sum_{i} R_{p i}^{V} A_{i}^{\mu}=\sum_{i} \frac{g_{i} \bar{\delta}_{i}^{p}}{\mu_{p}} A_{i}^{\mu}
$$

$(p=1 \ldots r)$. Note that the anomalous gauge boson masses (3.1. $\overline{1}$ i) depend on the blowing-up modes $m_{k}$ through the gauge couplings $g_{i}$ and the Kähler metric $K_{k l}$ (which appears in the couplings $\widetilde{\delta}_{i}^{k}$ ). But since the latter is non-singular at the orientifold point $\left\langle m_{k}\right\rangle=0$ (see below), they 
do not significantly depart from their orientifold value $\mu_{p} \sim M_{P l}$ when one blows up. We therefore conclude that the anomalous gauge bosons acquire Planck-scale masses through the GreenSchwarz mechanism while the $N-r$ anomalyfree gauge bosons remain massless, exactly as one would have expected.

As mentioned above, the Fayet-Iliopoulos terms are not generated at one loop (note that this is in agreement with the fact that the dilaton does not participate in anomaly cancellation), but receive tree-level contributions from the Kähler potential

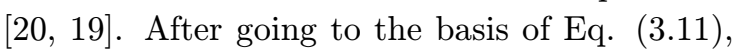
one obtains:

$$
\xi_{p}^{2}=-\frac{\mu_{p} M_{P l}}{\sqrt{2} g} \sum_{k, l} K_{k} K_{k l}^{-1 / 2} R_{p l}^{M}
$$

where $K_{k}=\partial K / \partial M_{k}$. Note that $\xi_{p}^{2}=0$ for anomaly-free $U(1)$ 's (since $\mu_{p}=0$ ). Expanding the derivatives of $K$ around the orientifold point $\left\langle m_{k}\right\rangle=0$, one finds:

$$
\xi_{p}^{2}=-\frac{\mu_{p} M_{P l}}{g}\left\langle m_{p}^{\prime}\right\rangle-\ldots
$$

To derive Eq. (13.15), we have used the fact that the Kähler potential (3.6.) can be expanded in powers of $\left(M_{k}+\bar{M}_{k}-2 \sum_{i} \delta_{i}^{k} V_{i}\right)$ around the orientifold point, and that the lowest order term is a square ${ }^{3}$. We conclude that the scale of the Fayet-Iliopoulos terms is set by the values of the blowing-up modes; in particular, they vanish in the orientifold limit. This is to be contrasted with the heterotic case, in which $\xi^{2}$ is tied to the string scale by the coefficient of the mixed gravitational anomaly.

To summarize, in $D=4, N=1$ type IIB orientifolds, the vector multiplets $V_{p=1 \ldots r}^{\prime}$ associated with the anomalous combinations of the abelian gauge factors acquire a Planck-scale mass (B. $\overline{3} . \overline{1}$ ) by absorbing a combination of the twisted chiral multiplets which contain the blowing-up modes of the underlying orbifold. To each of these massive multiplets is associated a modulidependent Fayet-Iliopoulos term (13.15 lowest order is proportional to the vev of its scalar component. After integrating out the massive

\footnotetext{
${ }^{3}$ This statement [19] is supported by the arguments of Ref. $[22]$, and has been confirmed by the computations of Ref. [21].
}

vector multiplets, we end up with $N-r$ anomalyfree $U(1)$ 's and $M-r$ twisted moduli $M_{p=r+1 \ldots M}^{\prime}$. Note that contrary to the heterotic case, a vacuum shift is not required to maintain supersymmetry. Indeed, at the level of unbroken supersymmetry, and in the absence of any nonperturbative mechanism that would stabilize them, the vevs of the $m_{k}$ fields are only restricted by the vanishing of the anomalous $D$-terms $(p=1 \ldots r)$

$$
D_{p}^{\prime}=-g\left(\sum_{\alpha} Y_{p}^{\prime \alpha}\left|\Phi_{\alpha}\right|^{2}+\xi_{p}^{2}\left(m_{k}\right)\right)
$$

Thus they describe flat directions, and the FayetIliopoulos terms in type IIB orientifolds are just moduli. In particular, there is an obvious vacuum $\left\langle\Phi_{\alpha}\right\rangle=0,\left\langle m_{k}\right\rangle=0$, corresponding to the orientifold limit, in which all $\xi_{p}^{2}$ vanish. In this vacuum, only the anomalous $U(1)$ 's are broken, and their associated gauge bosons become heavy and decouple, leaving $r$ residual global symmetries. On the other hand, nonzero values of the blowing-up modes would force some of the matter fields $\Phi_{\alpha}$ to acquire a vev, possibly leading to further breakdown of the gauge group ${ }^{4}$.

\section{Heterotic-type I duality in four dimensions}

$D=4, N=1$ type IIB orientifolds, which can be seen as orbifolds of the type I string theory, allow us to investigate four-dimensional aspects of heterotic-type I duality [1] ${ }_{1}^{n}$. Indeed, some of these models are expected to have heterotic duals which are perturbative string constructions. Furthermore, the duality relation between the tendimensional dilatons $\Phi_{I}^{(10)}=-\Phi_{H}^{(10)}$ becomes, upon reduction to $D$ dimensions [3in

$$
\Phi_{I}^{(D)}=\frac{6-D}{4} \Phi_{H}^{(D)}-\frac{D-2}{16} \ln V_{H}^{(10-D)}
$$

${ }^{4}$ Let us add for completeness that the combinations $\underline{M}_{k}+\bar{M}_{k}-2 \sum_{i} \delta_{i}^{k} V_{i}$ appearing in the Kähler potential (3.6. $)$ should actually be corrected by a piece depending logarithmically on the untwisted moduli $[23,121]$. Taking this effect into account does not modify our conclusions; in particular, the Fayet-Iliopoulos terms still vanish in the orientifold limit. 
where $V_{H}^{(10-D)}$ is the (10-D)-dimensional compact volume in heterotic string units. Thus, heterotic-type I duality, which is a nonperturbative symmetry in ten dimensions, relates regions of the moduli spaces which are both weakly coupled in four dimensions.

More specifically, among the consistent $T^{6} / Z_{N}$

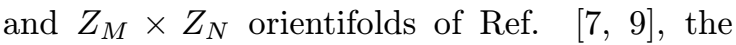
ones that contain solely D9-branes are expected to have a perturbative heterotic dual; these are the $Z_{3}, Z_{7}$ and $Z_{3} \times Z_{3}$ models. These models as well as their candidate heterotic duals were constructed in Ref. [3, pects were also discussed. However, the results of Ref. $\left[2 \overline{0}_{1}^{1}, \overline{1}, \overline{1} \overline{9}_{1}^{1}\right]$ were not known at that time (in particular, it was generally assumed that a FayetIliopoulos term of the order of the string scale is associated to each anomalous $U(1)$ ), which calls for a reexamination of the question. The purpose of this section is to perform a detailed investigation of duality between $D=4, N=1$ type IIB orientifolds and heterotic orbifolds on the basis of the results of previous section.

\section{1 $Z_{3}$ models}

Let us start with the $Z_{3}$ models of Ref. [3]. The orientifold model is constructed by gauging the world-sheet parity of the type IIB string compactified on $T^{6} / Z_{3}$. The gauge group is $G=$ $S U(12) \times S O(8) \times U(1)_{X}$, and the massless spectrum consists of 3 families of $Q_{a}=\left(\mathbf{1 2}, \mathbf{8}_{\mathbf{V}}\right)_{-\mathbf{1}}$ and $\Phi_{a}=(\overline{\mathbf{6 6}}, \mathbf{1})_{+\mathbf{2}}$ in the open string sector, 27 twisted moduli $M_{\alpha \beta \gamma}, \alpha, \beta, \gamma=1,2,3$ corresponding to the blowing-up modes of the underlying orbifold (which has $3^{3}=27$ fixed points) and 10 untwisted moduli (9 geometric moduli $T_{a b}$ and the dilaton) in the closed string sector. The renormalizable superpotential reads $W_{I}=$ $\lambda_{a b c}^{I} Q_{a} Q_{b} \Phi_{c}$. The $U(1)_{X}$ factor is anomalous, with non-universal anomalies $C_{S U(12)}=36, C_{S O(8)}$ $=-72, C_{X}=864$ and $\operatorname{Tr} X / 12=9$ which are canceled by the shifts of the twisted moduli $M_{\alpha \beta \gamma}{ }^{5}$.

Let us now consider the candidate heterotic dual, which is a $T^{6} / Z_{3}$ orbifold of the $S O(32)$ heterotic string. The gauge group is the same as

\footnotetext{
${ }^{5}$ More precisely, only the symmetric combination of the 27 twisted moduli $M_{\alpha \beta \gamma}$ is involved in the generalized Green-Schwarz mechanism
}

in the orientifold model, and the untwisted sector matches the open string and untwisted closed string sectors of the latter. The twisted sector, which contains 27 copies of $M_{\alpha \beta \gamma}=(\mathbf{1}, \mathbf{1})_{-4}$ and $V_{\alpha \beta \gamma}=\left(\mathbf{1}, \mathbf{8}_{\mathbf{S}}\right)_{+\mathbf{2}}$, shows on the other hand strong differences with the orientifold model: the blowing-up modes $M_{\alpha \beta \gamma}$ are charged under $U(1)_{X}$ and the $S O(8)$ spinors have no orientifold counterparts (this is due to the fact that spinorial representations of orthogonal groups cannot arise perturbatively from $D$-branes; however, they could be generated nonperturbatively). The renormalizable superpotential differs from $W_{I}$ by the presence of couplings involving the twisted fields [4]" $W_{H}=\lambda_{a b c}^{H} Q_{a} Q_{b} \Phi_{c}+\Lambda_{(\ldots)} M_{\alpha \beta \gamma} V_{\alpha^{\prime} \beta^{\prime} \gamma^{\prime}} V_{\alpha^{\prime \prime} \beta^{\prime \prime} \gamma^{\prime \prime}}$. Finally, the $U(1)_{X}$ factor has universal anomalies $C_{S U(12)}=C_{S O(8)}=C_{X}=\operatorname{Tr} X / 12=36$ which are compensated for by the shift of the dilaton, and a Fayet-Iliopoulos term $\xi^{2}=\frac{\operatorname{Tr} X}{192 \pi^{2}} M_{S t r}^{2}$ is generated at the one-loop level.

Although the two models appear to be quite different, the presence of an anomalous $U(1)$ on both sides enforces duality. Indeed, one should compare not the original vacua, but the supersymmetric low-energy theories which are obtained after the anomalous $D$-term has been canceled and the heavy fields (including the anomalous $U(1))$ have been integrated out. On the orientifold side, the vector multiplet $V_{X}$ becomes massive by absorbing the symmetric combination of the 27 twisted moduli $M_{\alpha \beta \gamma}$; its decoupling leads to an unbroken gauge group $S U(12) \times S O(8)$ and a global symmetry $U(1)_{X}$ at the orientifold point. On the heterotic side, a vacuum shift is necessary in order to compensate for the FayetIliopoulos term. Since the latter is positive, this requires nonzero vevs of the blowing-up modes, which carry a negative $X$-charge ${ }^{6}$. The twisted states $V_{\alpha \beta \gamma}$ then acquire supersymmetric masses through their superpotential couplings [4ind] and decouple from the massless spectrum. As long as the $\left\langle M_{\alpha \beta \gamma}\right\rangle$ are the only nonzero vevs, the non abelian gauge group $S U(12) \times S O(8)$ remains un-

\footnotetext{
${ }^{6}$ Since the $Q_{a}$ fields also carry a negative $X$-charge, one can actually cancel the anomalous $D$-term without blowing up the orbifold. We do not consider this possibility however, because it seems to be impossible to ensure both $D$ - and $F$-flatness in this case (a similar statement has been done about the blown-up orientifold [ [24 $\left.\mathbf{2}^{1}\right]$ ).
} 
broken, while $V_{X}$ becomes heavy by absorbing a combination of the dilaton and $M_{\alpha \beta \gamma}$ superfields. Note that $U(1)_{X}$ survives as an effective global symmetry of the renormalizable superpotential, although it is spontaneously broken by the vevs of the blowing-up modes. This is due to the fact that the only renormalizable couplings that violate the $X$ charge are the masses of the $V_{\alpha \beta \gamma}$ fields, which decouple from the low-energy theory. Of course, higher order terms may not respect this symmetry.

We conclude that the gauge groups and massless spectra of both models match after decoupling of the anomalous $U(1)$. It is interesting to remark that, at the point of maximal gauge symmetry, the blown-up orbifold is dual to the type I model in the exact orientifold limit. Note also that the presence of moduli-dependent Fayet-Iliopoulos terms on the orientifold side is crucial for matching all flat directions of both models.

\subsection{1 $Z_{3}$ models with a discrete Wilson line}

One can add a discrete Wilson line to the $Z_{3}$ models [8]. The gauge group is then broken to $G=S U(4)^{4} \times U(1)^{4}$. Three combinations $X, Y_{1}$ and $Y_{2}$ of the abelian charges are anomalous in the orientifold model, thus only one $U(1)$ gauge factor remains at low energy. On the heterotic side, there is of course a single anomalous charge $X$, but the vacuum shift induced by the FayetIliopoulos term breaks $Y_{1}$ and $Y_{2}$ at the same time. Finally, one finds a perfect matching of both models once the heavy fields have been integrated out.

\section{$4.2 Z_{7}$ models}

While duality holds in the $Z_{3}$ case, problems appear at the level of the maximal unbroken gauge symmetry in the $Z_{7}$ case. These models a gauge group $G=S U(4)^{3} \times S O(8) \times U(1)^{3}$, with three anomalous $U(1)$ 's on the orientifold side. The heterotic model has $\xi^{2}>0$ and the only fields with a negative anomalous charge have quantum numbers $Q_{1}=(\mathbf{1}, \overline{\mathbf{4}}, \mathbf{4}, \mathbf{1})_{(-\mathbf{2}, \mathbf{1}, \mathbf{1})}, Q_{2}=$ $(\overline{\mathbf{4}}, \mathbf{1}, \mathbf{4}, \mathbf{1})_{(-\mathbf{2}, \mathbf{1},-\mathbf{1})}$ and $Q_{3}=(\overline{\mathbf{4}}, \overline{\mathbf{4}}, \mathbf{1}, \mathbf{1})_{(-\mathbf{2},-2,0)}$ (where the first subscript refers to the anomalous charge). Thus the vacuum shift necessarily breaks $S U(4)^{3}$ together with the three $U(1)$ 's. In the orientifold model, on the other hand, the non abelian gauge group remains unbroken as long as the singularities are not blown up, since the Fayet-Iliopoulos terms vanish in this limit. We conclude that the moduli spaces of the two models cannot be matched, unless the orientifold point is destabilized e.g. by some nonperturbative mechanism ${ }^{7}$.

\section{$4.3 Z_{3} \times Z_{3}$ models}

Duality does not seem to hold in the $Z_{3} \times Z_{3}$ case either. Both models $[\overline{6}|\overline{1}|$ have a gauge group $G=$ $S U(4)^{3} \times S O(8) \times U(1)^{3}$ with a single anomalous $U(1)$. After the vacuum shift and the decoupling of the heavy fields, the gauge groups match, but there remains a slight discrepancy in the massless spectrum: some heterotic twisted states are charged under the two remaining $U(1)$ 's, while their orientifold counterparts are gauge singlets.

\subsection{Comments}

It is difficult to draw a general conclusion from the previous analyses. While the $Z_{3}$ models satisfy our duality criteria, problems appear at the level of the gauge group in the $Z_{7}$ case, and at the level of the massless spectrum in the $Z_{3} \times Z_{3}$ case. It could be that these models are actually not dual. However, the discrepancies found could just mean that we are missing nonperturbative effects; in particular, duality could be restored in the $Z_{7}$ case if Fayet-Iliopoulos terms were generated in the orientifold model.

That such effects may be present is supported by several features of the $Z_{3}$ and $Z_{3} \times Z_{3}$ models that strongly suggest duality. First, despite the differences of the anomaly cancellation mechanisms, the blowing-up modes of the two models seem to play a "dual" role: on the orientifold side, they are moduli and participate in the generalized Green-Schwarz mechanism; on the heterotic side, they are charged under the anomalous $U(1)$ and acquire vevs in order to compensate for the Fayet-Iliopoulos term. This results

\footnotetext{
${ }^{7}$ Let us add for completeness that the $Z_{7}$ orbifold also possesses charged twisted states that have no perturbative counterparts on the orientifold side. Like in the $Z_{3}$ case, these states can acquire large masses through their superpotential couplings. However, in the $Z_{7}$ case this requires nonzero vevs of both the $Q_{a}$ fields and the blowing-up modes, the latter being not enforced by the vacuum shift.
} 
in the breaking and decoupling at a high scale of the same number of $U(1)$ 's in both models. Furthermore, the vevs of the heterotic blowingup modes give large supersymmetric masses to the states that have no perturbative orientifold counterparts, making it possible for both massless spectra to match. This picture is not valid for the $Z_{7}$ models, because the heterotic blowingup modes do not carry any anomalous charge.

\section{Conclusion}

In this talk, we have studied the properties of anomalous $U(1)$ 's in a large class of $D=4, N=1$ type IIB orientifolds, and reconsidered some candidate evidence for $N=1$ heterotic-type I duality in four dimensions, in the light of the results of Ref. [200 and [1 190 . We have shown how the anomalous vector multiplets acquire a mass of the order of the Planck scale and decouple from the low-energy effective theory. The scales of the associated Fayet-Iliopoulos terms are fixed by the values of the blowing-up modes of the underlying orbifold, and they vanish in the orientifold limit. This is a noticeable difference with the heterotic anomalous $U(1)$, whose Fayet-Iliopoulos term has a nonzero, moduli-independent value of the order of the string scale.

On the basis of this picture, we have reconsidered the generally admitted duality between $D=4, N=1$ type IIB orientifolds and heterotic orbifolds, which has been considered as a four-dimensional manifestation of the postulated heterotic-type I duality in ten dimensions. We found that the shift of the heterotic vacuum required by the presence of a Fayet-Iliopoulos term ensures a perfect matching of the gauge groups and perturbative spectra of the $Z_{3}$ models, but there remain discrepancies in two other candidate dual models, namely the $Z_{7}$ and $Z_{3} \times Z_{3}$ cases. It is likely however that duality be restored by nonperturbative effects that remain to be identified ${ }^{8}$.

\footnotetext{
${ }^{8}$ The results of Ref. $[21]$, in which threshold corrections to the gauge couplings in $T^{6} / Z_{N}$ type IIB orientifolds were computed, seem to indicate that such nonperturbative effects are also required in the $Z_{3}$ case in order for duality to hold.
}

\section{Acknowledgements}

I would like to thank Z. Lalak and H.P. Nilles for a pleasant and stimulating collaboration. This work has been supported by the European Commission program ERBFMRX-CT96-0090.

\section{References}

[1] Z. Lalak, S. Lavignac and H.P. Nilles, preprint BONN-TH-99-06, hep-th/9903160, to appear in Nucl. Phys. B.

[2] A. Sagnotti, in Cargese '87, "Non-Perturbative Quantum Field Theory", eds. G. Mack et al. (Pergamon Press, Oxford, 1988), p. 251; G. Pradisi and A. Sagnotti, Phys. Lett. B216 (1989) 59; M. Bianchi and A. Sagnotti, Phys. Lett. B247 (1990) 517, Nucl. Phys. B361 (1991) 519; E.G. Gimon and J. Polchinski, Phys. Rev. D54 (1996) 1667.

[3] C. Angelantonj, M. Bianchi, G. Pradisi, A. Sagnotti, and Ya.S. Stanev, Phys. Lett. B385 (1996) 96.

[4] Z. Kakushadze, Nucl. Phys. B512 (1998) 221.

[5] Z. Kakushadze and G. Shiu, Phys. Rev. D56 (1997) 3686.

[6] Z. Kakushadze and G. Shiu, Nucl. Phys. B520 (1998) 75 .

[7] G. Zwart, Nucl. Phys. B526 (1998) 378.

[8] L.E. Ibanez, JHEP 07 (1998) 002.

[9] G. Aldazabal, A. Font, L.E. Ibanez, and G. Violero, Nucl. Phys. B536 (1998) 29.

[10] Z. Kakushadze, Phys. Lett. B434 (1998) 269, Phys. Rev. D58 (1998) 101901.

[11] J. Lykken, E. Poppitz and S.P. Trivedi, Nucl. Phys. B543 (1999) 105.

[12] L.E. Ibanez, C. Munoz and S. Rigolin, Nucl. Phys. B553 (1999) 43

[13] J. Polchinski and E. Witten, Nucl. Phys. B460 (1996) 525.

[14] M. Dine, N. Seiberg and E. Witten, Nucl. Phys. B289 (1987) 317.

[15] M. Green and J. Schwarz, Phys. Lett. B149 (1984) 117.

[16] J. Atick, L. Dixon and A. Sen, Nucl. Phys. B292 (1987) 109; M. Dine, I. Ichinose and N. Seiberg, Nucl. Phys. B293 (1987) 253. 
[17] A. Font, L.E. Ibanez, H.P. Nilles and F. Quevedo, Nucl. Phys. B307 (1988) 109; ibid., B310 (1988) 764; J.A. Casas, E.K. Katehou and C. Munoz, Nucl. Phys. B317 (1989) 171.

[18] A. Sagnotti, Phys. Lett. B294 (1992) 196.

[19] E. Poppitz, Nucl. Phys. B542 (1999) 31.

[20] L.E. Ibanez, R. Rabadan and A.M. Uranga, Nucl. Phys. B542 (1999) 112.

[21] I. Antoniadis, C. Bachas and E. Dudas, preprint CPHT-S714.0399, LPT-ORSAY 99/12, hepth/9906039.

[22] M.R. Douglas and G. Moore, preprint RU-9615, hep-th/9603167.

[23] L.E. Ibanez, R. Rabadan and A.M. Uranga, preprint FTUAM-99-14, hep-th/9905098.

[24] M. Cvetic, L. Everett, P. Langacker and J. Wang, JHEP 04 (1999) 020. 